\title{
An extension of Newton-Raphson power flow problem
}

\author{
Mevludin Glavic ${ }^{\mathrm{a}, *}$, Fernando L. Alvarado ${ }^{\mathrm{b}}$ \\ ${ }^{a}$ University of Liege, Electrical Engineering and Computer Science Department, Sart-Tilman B28, B-4000 Liege, Belgium \\ ${ }^{\mathrm{b}}$ University of Wisconsin-Madison, Electrical and Computer Engineering Department, Madison, WI 53706, USA
}

\begin{abstract}
This paper explores an idea to extend Newton-Raphson power flow problem to handle power system transmission line flow limits, by means of generation redispatch and phase shifters. We extend and reformulate the power flow so that it includes a variety of flow limits (thermal, small-signal stability, voltage difference), generation redispatch, and phase shifters. The novelty of the approach is three step procedure (in case any limit violations exist in the system): run ordinary power flow (and identify flow limits violated), solve a set of linear equations using extended power flow Jacobian by adding a new column and a new raw that characterize particular limit, and resolve ordinary power flow with initial solution obtained after the correction made by solution of linear equations. The use of ordinary power flow Jacobian and minimal extensions to it in the case of limits identified makes this approach an attractive alternative for practical use. A simple numerical example and the examples using an approximate model of real-life European Interconnected Power System are included in the paper to illustrate the concept.
\end{abstract}

(c) 2006 Elsevier Inc. All rights reserved.

Keywords: Newton-Raphson power flow; Power system; Generator redispatch; Phase shifters; Flow limits

\section{Introduction}

The steady-state conditions of a power system are typically modeled, from Kirchoff laws and power conservation, by a system of non-linear algebraic equations [1-3]:

$$
\mathbf{f}(\mathbf{x})=\mathbf{0}
$$

where $\mathbf{x} \in \mathfrak{R}^{n}$ is the vector of state variables (bus voltage magnitudes and phase angles), and $\mathbf{f}: \mathfrak{R}^{n} \rightarrow \mathfrak{R}$ is sufficiently smooth function. Ordinary power flow problem [1,2] for a system with $N$ buses (out of which $N_{\mathrm{g}}$ are generation buses) consists of the solution of $n=N+\left(N-N_{\mathrm{g}}+1\right)$ simultaneous non-linear equations with $n$ unknowns. The equations that are solved simultaneously include: an active power balance equation $\Delta P_{i}$ for

\footnotetext{
* Corresponding author.

E-mail address: glavic@montefiore.ulg.ac.be (M. Glavic).

$U R L$ : http://www.montefiore.ulg.ac.be/ glavic (M. Glavic).
} 


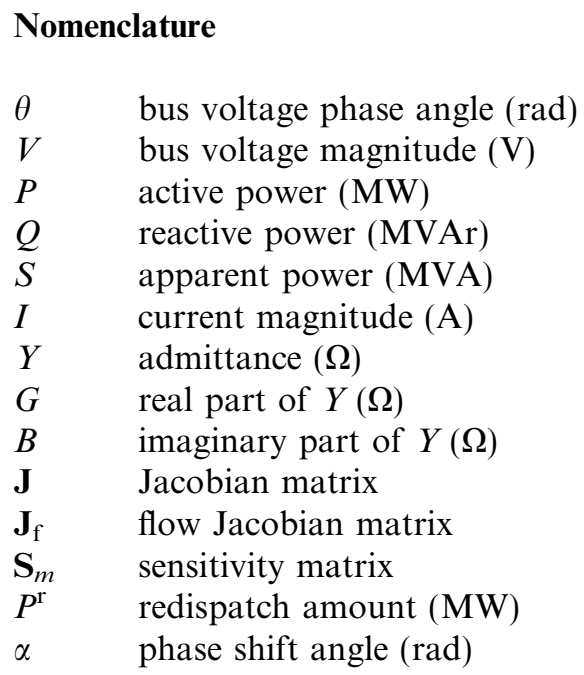

every bus except one bus that is designated as the "slack" bus (the bus so designated is always one of the generation buses), and a reactive power balance equation $\Delta Q_{i}$ for every load bus (buses where both $P$ and $Q$ injections are specified). There are many extensions of the ordinary power flow problem aiming either to ease computational burden or to include some realistic limits such as power generation (both active and reactive power) or bus voltage magnitude limits $[1,2]$. Consideration of realistic physical power system limits is of paramount importance in order to ensure physical feasibility of the solution.

The factors that influence the limiting values of transmission line flows are [4]: thermal limit $\left(I_{\mathrm{f}}^{2}\right.$ limit, $I$ stands for current while $\mathrm{f}$ stands for flow), small-signal stability limit ( $P_{\mathrm{f}}$ limit), and voltage difference limit ( $S_{\mathrm{f}}$ limit, $S$ stands for apparent power). Both generation redispatch and phase shifters have been recognized as useful means to handle line flow limits. For some early considerations interested readers are referred to [5-9] and for recent ones to [10-13]. All these considerations could be roughly classified as optimization $[8,13]$ and non-optimization (direct) based [5-7,9-12]. Optimization based methods (particularly optimal power flow) are arguably more accurate but computationally expensive and time consuming [11]. Direct methods emerged as the need for an efficient and fast method that trade optimality for effectiveness, so that power system operators can make quick yet efficient decisions under stressed conditions of the power system. The method developed in this paper belong to direct methods and differs from previous similar considerations in the way how ordinary power flow is extended and reformulated in order to include a variety of flow limits, generation redispatch and phase shifters. The novelty of the approach is three step procedure (in case any limit violations exist in the system): run ordinary power flow (and identify flow limits violated), solve a set of linear equations using extended power flow Jacobian by adding a new column and a new raw that characterize particular limit, and resolve ordinary power flow with initial solution obtained after the correction made by solution of linear equations. The use of ordinary power flow Jacobian and minimal extensions to it in the case of limits identified makes this approach an attractive alternative for practical use. To facilitate the presentation throughout of the paper we focus on active power flow limits and in the appendix provide generic derivations for all three types of line flow limits. We extend the fast vectorized version of ordinary power flow, implemented in MATLAB ${ }^{\circledR}$ environment $[2,3]$.

The paper is organized as follows. In Section 2 ordinary power flow problem is reviewed and possible extensions discussed, Section 3 describes how flow limits can be relieved by generation redispatch and provides some analytical results on the reformulation of ordinary power flow problem. Possibilities of using phase-shifting transformers for the same purpose are presented in Section 4. Section 5 provides the results obtained with help of real-life European Interconnected power system, while Section 6 offers some conclusions. 


\section{Ordinary power flow and approaches to handle flow limits}

The power flow equations are given by [1-3]

$$
\mathbf{f}(\mathbf{x})=\left(\begin{array}{c}
\Delta \mathbf{P}(\mathbf{x}) \\
\Delta \mathbf{Q}(\mathbf{x})
\end{array}\right)=\mathbf{0}
$$

where $\mathbf{x}=\left(\theta^{\mathrm{T}}, V^{\mathrm{T}}\right)^{\mathrm{T}} \in \mathfrak{R}^{n}$ is the state vector representing the bus voltage angle $(\theta)$ and magnitude $(V)$.

The Newton-Raphson is usually the method of choice to solve this system of non-linear equations. Starting from a good initial guess $\left(\mathbf{x}_{0}\right)$ this method determines the solution through iterative scheme,

$$
\begin{aligned}
& {\left[\begin{array}{c}
\boldsymbol{\Delta} \mathbf{P}^{k} \\
\boldsymbol{\Delta} \mathbf{Q}^{k}
\end{array}\right]=\underbrace{\left[\begin{array}{ll}
\mathbf{H}^{k} & \mathbf{N}^{k} \\
\mathbf{M}^{k} & \mathbf{L}^{k}
\end{array}\right]}_{\mathbf{J}} \times\left[\begin{array}{c}
\boldsymbol{\Delta} \boldsymbol{\theta}^{k} \\
\boldsymbol{\Delta} \mathbf{V}^{k}
\end{array}\right],} \\
& \boldsymbol{\theta}^{k+1}=\boldsymbol{\theta}^{k}+\boldsymbol{\Delta} \boldsymbol{\theta}^{k}, \\
& \mathbf{V}^{k+1}=\mathbf{V}^{k}+\boldsymbol{\Delta} \mathbf{V}^{k},
\end{aligned}
$$

where $\boldsymbol{\theta}^{0}=\boldsymbol{\theta}_{0}, \mathbf{V}^{0}=\mathbf{V}_{0}$, and $\mathbf{J}$ is the Jacobian matrix. Newton-Raphson method will converge quadratically from a sufficiently good initial guess, provided that the Jacobian is non-singular at the solution [1,2]. After the initial solution (the phase angle at every bus in the system except one that is designated as the "reference" bus and the voltage magnitude at every load bus in the system) is performed, one then can solve for every other, derived, quantity of interest in the system. Having calculated all derived quantities, one can then verify whether they are within their acceptable range of values. The power flow problem becomes somewhat more complicated when one is interested in either adding or removing degrees of freedom (or variables) to the basic problem. There are many reasons for adding and/or removing one or more degrees of freedom, but for purpose of this paper we are interested in one when the flow on a particular line or corridor exceeds its designated capability.

Resolution of the flow limits problem can be done in many ways, including (a) ignoring and reporting the problem, (b) adjusting the injections of the generators to resolve the problem, (c) adjusting the setting of some transformer, (d) phase shifter or phase angle regulator) to control the flow, (e) adjust the reactive injections to reduce the current or the MVAs (Mega-Volt-Amperes) on the offending circuit without necessarily adjusting the active power injections, or (f) simply tripping or removing the line from the service. Method (f) is surprisingly effective in many situations, particularly when the offending line is a low-voltage or low-capacity line. For purpose of this paper, however, we will consider the use of methods (b) and (c) and will examine the impact on the power flow formulation and solution techniques of using these methods.

\section{Relieving flow limits by generation rescheduling}

The problem at hand is stated quite simply: extend the formulation and solutions method of the power flow so that when the flow on a given line (or on any line or set of lines, for that matter) exceeds some specified limit, generation is redispatched just until the point where the limit is no longer violated. The selection of the redispatch unit or units can be done in many ways. We specifically consider four ways in which this can be done:

- The user (the power system operator) has predesignated which generators are to be redispatched, either for all constraints or, better yet, for every possible constraint the user has specified the corresponding generation pair (yes, it mast be a pair) of generators that are to be redispatched. If only one generator is designated for redispatch, it is implicitly assumed that the slack bus is the other generator in the pair. We call this type of redispatch "User (operator) specified".

- The program is to determine the generator pair using a "most effective" criterion. That is, the generator pair that will have the maximum impact on limiting the flow with the minimum amount of redispatch (at least in a linearized sense) is designated as the generator pair of interest. This type of redispatch is referred to as "Most effective". 
- The program is to determine the generator pair using a "maximum margin" criterion. That is, the generators that are capable of doing the redispatch with a minimum percentage impact to their available limits are used for the purpose. We call this type of redispatch "Sufficient".

- The program determines the generator pair based on a "minimum redispatch cost criterion (this assumes that marginal costs are known). This type of redispatch is termed as "Cheapest".

All of these redispatch versions can be implemented with or without penalty factors (that is, active power system losses can either be considered or be ignored during these calculations). For the sake of simplicity, only lossless case is actually illustrated.

Further we consider two options for the "operator-specified" redispatch: "Chunk" and "Continuous". In "Chunk" option the operator simply specifies a generator pair and the amount of active power to be redispatched. In "Continuous" redispatch the operator specifies a generator pair, but not the amount of active power to be redispatched which is to be solved. "Most effective" redispatch is eminently technical. The choice of a proper generator pair is based on sensitivities of the line flow in relation to each system generator.

"Sufficient" and "Cheapest" redispatch also rely on sensitivity approach and a proper generator pair for "sufficient" and "cheapest" redispatch is chosen according to the next formulation,

$$
\begin{aligned}
& \text { "Sufficient" }=\text { "Mosteffective" } \times\left(P_{\max }-P_{\text {actual }}\right) \text { for 'INC', } \\
& \text { "Sufficient" }=\text { "Mosteffective" } \times\left(P_{\text {actual }}-P_{\min }\right) \text { for 'DEC', } \\
& \text { "Cheapest" = "Sufficient" } \times \text { MU for both,'INC' and 'DEC', }
\end{aligned}
$$

where "INC" stands for increase, "DEC" for decrease, and MU represents the cost of generation in terms of a monetary unit.

\subsection{Finding the most effective generator pair}

The problem at hand is to find the generator pair that has the greatest impact on a particular line flow. In a linearized sense, the impact of a generator pair redispatch on a given flow can be computed as the impact of two separate changes: the impact of an injection at one bus (the bus that will increase its generation) and the impact of a decrease of power injection at another bus (the bus that will decrease its generation). In either of these cases the changes are absorbed by the slack bus. Handling of losses can be done either by ensuring that the changes have no impact on the injection at the slack bus (this requires the use of penalty factors) or by letting the slack bus absorb any changes in losses. To avoid introducing additional issues, we elect the latter option here.

The equations for the determining of sensitivities of flows are best defined by expressing the flows on all lines of interest in terms of the fundamental variables $V$ and $\theta$. We first consider only "sending end" flows, but it would be equally simple to consider "receiving end" flows (sending and receiving ends are two sides of a transmission line). We assume that the limits of interest are expressed in terms of active power. It is relatively straightforward to consider a current or a MVA limit (for details see Appendix A). To facilitate the expression of flow equations, we introduce the concept of a flow admittance matrix, $\overline{\mathbf{Y}}_{\mathrm{f}}$. Typically, this matrix has only two nonzero entries per row, one at the sending end and one at the receiving end of each branch. However, it can be generalized to express the sum of flows across any set of branches. In this case, it will have additional entries. This is a matrix that, when multiplied by the vector of complex voltages $\overline{\mathbf{V}}$, yields the vector of sending end current $\overline{\mathbf{I}}_{\mathrm{f}}$ for all branches of interest in the system. It is possible to create such a matrix even in cases where $\pi$-equivalents of lines are used or when we are dealing with tap-changing phase-shifting transformers. The vector of complex sending end voltages is denoted by $\mathbf{V}^{\text {from }}$. Thus, in complex form

$$
\overline{\mathbf{S}}_{\mathrm{f}}=\operatorname{diag}\left(\overline{\mathbf{V}}^{\text {from }}\right) \operatorname{conj}\left(\overline{\mathbf{Y}}_{\mathrm{f}} \overline{\mathbf{V}}^{\text {from }}\right) .
$$

Let $V_{k}$ denote the voltage magnitude at the from end of branch $k$, and $\theta_{k i}$ denote $\theta_{k}-\theta_{i}$. From here, the expression for any flow $k$ on a branch can be determined from 


$$
\bar{S}_{k}^{\text {from }}=\sum_{i \in\left\{i \mid \bar{Y}_{\mathrm{f}}^{k i} \neq 0\right\}} V_{k} V_{i} \angle \theta_{k i}\left(G_{k i}-\mathrm{j} B_{k i}\right),
$$

where $G_{k i}$ and $B_{k i}$ are the real and imaginary parts of the admittance between the buses $k$ and $i$.

The summation generally has just two terms. To find the real part of this expression we use

$$
P_{k}^{\text {from }}=\sum_{i \in\left\{i \mid \bar{Y}_{\mathrm{f}}^{k i} \neq 0\right\}}\left(V_{k} V_{i} G_{k i} \cos \theta_{k i}+V_{k} V_{i} B_{k i} \sin \theta_{k i}\right) .
$$

This summation also generally has only two terms, one for each end of the branch in question.

In order to find the sensitivity of a branch flow $P_{k}^{\text {from }}$ to a change in any bus injection, we first determine the sensitivity of the branch flow with respect to changes in voltages and angles, and separately we determine the sensitivity of the voltages and angles to change in injection. This last calculation is done using an ordinary power flow Jacobian matrix. That is, we determine

$$
\Delta \mathbf{P}_{\mathrm{f}}=\mathbf{J}_{\mathrm{f}}\left[\begin{array}{c}
\Delta \boldsymbol{\theta} \\
\Delta \mathbf{V}
\end{array}\right]
$$

where

$$
\mathbf{J}\left[\begin{array}{c}
\Delta \theta \\
\Delta \mathbf{V}
\end{array}\right]=\left[\begin{array}{c}
\Delta \mathbf{P} \\
\Delta \mathbf{Q}
\end{array}\right] .
$$

Here $\Delta \mathbf{P}$ is a vector with a single nonzero, at the location of the desired injection or extraction. Every location in the system can, of course, be considered a candidate location, which requires one computation per candidate location. Although in practice only generator locations may be of interest, the calculation above requires the use of the complete power flow Jacobian, however. The right hand side $\Delta \mathbf{Q}$ is zero in this transformation, since we are considering only changes in active power injections. $\mathbf{J}$ is the ordinary power flow Jacobian. $\mathbf{J}_{\mathrm{f}}$ is the Jacobian of the flow equations with respect to the voltages and angles (details below). Thus, obtaining the sensitivity of flows with respect to individual injections according to this explanation performs one repeat solution per location, and a single matrix vector multiplication yields all the sensitivities for all the lines of interest. The flow Jacobian matrix $\mathbf{J}_{\mathrm{f}}$ can be organized into its derivatives with respect to voltage angles (denoted by a submatrix $\mathbf{H}_{\mathrm{f}}$ ) and its derivatives with respect to voltage magnitudes (denoted by a submatrix $\mathbf{N}_{\mathrm{f}}$ ). Only sensitivities with of active power flows are considered here. The expressions for the entries of these submatrices are

$$
\begin{aligned}
& H_{\mathrm{f}}^{k i}= \begin{cases}0 & \text { if } Y_{\mathrm{f}}^{k i}=0, \\
-V_{k} V_{i} G_{k i} \sin \theta_{k i}+V_{k} V_{i} \cos \theta_{k i} & \text { if } k \neq i, \\
2 V_{k} G_{k i} & \text { if } k=i .\end{cases} \\
& N_{\mathrm{f}}^{k i}= \begin{cases}0 & \text { if } Y_{\mathrm{f}}^{k i}=0, \\
V_{k} G_{k i} \cos \theta_{k i}+V_{k} \sin \theta_{k i} & \text { if } k \neq i, \\
2 V_{k} G_{k i} & \text { if } k=i .\end{cases}
\end{aligned}
$$

From these expressions, one can then compute the sensitivity matrix $\mathbf{S}_{m}$. The dimension of this matrix is initially the number of line flows of interest $m$ by the number of degrees of freedom in the power flow. This matrix can be expanded to include explicitly all $n$ active power injections and all $n$ reactive power injections. This is done simply by padding the matrix with zero-valued columns for all such locations. This is so because an injection of power at the slack location produces no flow changes in the system, since it is absorbed by a corresponding injection at the same bus. Likewise for reactive power injections at all generation buses that are actively controlling the voltage.

Of interest to us is the use of a single row of $\mathbf{S}_{m}$, the row that corresponds to the location of a line that happens to be limiting (at or above its flow limit). We assume that there is only one such line. If the matrix has been expanded to include the reference location as indicated above, let $\mathbf{s}$ denote the first $n$ entries of the desired row of $\mathbf{S}_{m}$. The meaning of these entries is the impact on the flow on the limiting line of an injection at every point in the system. Only the subset of entries of $\mathbf{s}$ that correspond to generation locations is of actual interest, according to our rules. Denote this subvector by $\mathbf{s}^{\mathrm{g}}$. The largest-valued entry in $\mathbf{s}^{\mathrm{g}}$ identifies the system generator where a generator would have the greatest positive impact on the line flow of interest. The smallest-valued entry 
(most negative) in $\mathbf{s}^{\mathrm{g}}$ identifies the system generator where a generator would have the greatest adverse impact on the flow (that is, where a decrease in generation would have the greatest value in reducing the flow). It is quite possible that no generator has a negative or a positive value. In this case, the best generator in either direction is the reference generator. The determination of the best generator pair for redispatch purposes is thus very simple:

- Determine the vector $\mathbf{s}^{\mathrm{g}}$ of sensitivity of flows to injections for all generators in the system (or at least the eligible subset).

- Sort this vector.

- Select the largest-valued location $M$.

- Select the smallest-valued location $m$.

\subsection{Power flow with flow limit and generation redispatch}

Having determined a location of the pair of generators to redispatch $m, M$, next step is to formulate a modified ordinary power flow problem. The modifications required are as follows:

- Add an additional degree of freedom (or variable) called the "redispatch amount" $P^{\mathrm{r}}$.

- The equation for active power balance for node $m$ gets modified by the addition of $P^{\mathrm{r}}$.

- The equation for active power balance for node $M$ gets modified by the subtraction of $P^{\mathrm{r}}$.

- A new equation is added to the equation set. This equation specifies precisely the flow at the from end of the constrained line.

- The Jacobian gets modified by the inclusion of one additional column of all zeros except for one +1 in the active power row corresponding to $\mathrm{m}$ and one -1 in the active power row corresponding to $M$ (if either $m$ or $M$ corresponds to the slack bus, there is no entry added to the new Jacobian column).

- The Jacobian gets further modified by the inclusion of the Jacobian row for the added power flow restriction equation. This row has no more than four nonzero elements.

In the end, a single row and a single column get added to the Jacobian and a new slightly larger problem

$$
\left[\begin{array}{c}
\Delta \mathbf{P} \\
\Delta \mathbf{Q} \\
\hdashline \mathrm{rhs}
\end{array}\right]=\left[\begin{array}{cc:c}
\mathbf{H} & \mathbf{N} & \mathbf{k 1} \\
\mathbf{M} & \mathbf{L} & \mathbf{p} \\
\hdashline \mathbf{F F} 1 & 0
\end{array}\right] \times\left[\begin{array}{c}
\Delta \boldsymbol{\theta} \\
\boldsymbol{\Delta} \mathbf{V} \\
\hdashline P^{\mathrm{r}}
\end{array}\right]
$$

is to be solved (rhs stands for right-hand side). If two limits are identified one more column and a raw are to be added

$$
\left[\begin{array}{c}
\Delta \mathbf{P} \\
\boldsymbol{\Delta} \mathbf{Q} \\
\hdashline \text { rhs1 } \\
\hdashline \text { rhs2 }
\end{array}\right]=\left[\begin{array}{cc:c:c}
\mathbf{H} & \mathbf{N} & \mathbf{k 1} & \\
\mathbf{M} & \mathbf{L} & & \mathbf{k 2} \\
\hdashline \mathbf{F F} 1 & & 0 & \\
\hdashline & \mathbf{F F} 2 & 0
\end{array}\right] \times\left[\begin{array}{c}
\Delta \boldsymbol{\theta} \\
\boldsymbol{\Delta} \mathbf{V} \\
\hdashline P^{\mathrm{r} 1} \\
\hdashline P^{\mathrm{r} 2}
\end{array}\right]
$$

Every new limit adds a new column and a new raw. In practice, the number of transmission lines that are limited at the same time is not big (otherwise, huge number of limits indicate heavily stressed power system conditions for which the model of the system in form of differential-algebraic equations is appropriate mathematical description of the system). Moreover, the proposed extension fits well into problem of maximum system loadability determination either by repetitive solution of the power flow problem or by using a continuation power flow formulation in which the extension could be used in corrector step. Incremental increase of the system load during maximum loadability determination usually results in one limit violation at the time $[4,12]$.

The new entries in the Jacobian matrix will not create any fill-ins in the matrix factorization (sparsity of the Jacobian matrix is preserved what is of paramount importance for realistically sized power systems that might include thousands of buses). 


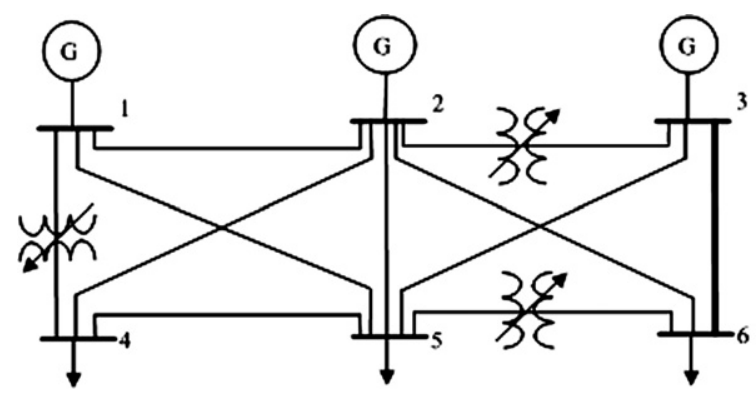

Fig. 1. One-line diagram of 6-bus system [1].

\subsection{A simple numerical example}

All these concepts are best explained by means of a simple numerical example. Because additional degrees of freedom are essential to make the problem non-trivial, we will consider for this example the 6-bus system illustrated in Fig. 1. This system is a slight variant of the example in [1]. Active power flow limit is considered in this example.

The "flow Jacobian" relating the active power flow on the limited line (line between buses 3 and 6 , shown in bold in Fig. 1, the excess of the overload is $3.5 \mathrm{MW}$, MegaWatts) to all six bus angles and six voltage magnitudes is given by

$$
\begin{aligned}
& \frac{\mathrm{d} P_{36}}{\mathrm{~d} \theta}=\left[\begin{array}{llllll}
0 & 0 & 10.3261 & 0 & 0 & -10.3261
\end{array}\right], \\
& \frac{\mathrm{d} P_{36}}{\mathrm{~d} V}=\left[\begin{array}{llllll}
0 & 0 & 2.4639 & 0 & 0 & -1.7698
\end{array}\right] .
\end{aligned}
$$

The sensitivity of flow on the limited line with respect to active and reactive power injections is given by

$$
\left[\begin{array}{l}
\Delta P_{2} \\
\Delta P_{3} \\
\Delta P_{4} \\
\Delta P_{5} \\
\Delta P_{6} \\
\Delta Q_{4} \\
\Delta Q_{5} \\
\Delta Q_{6}
\end{array}\right]=\left[\begin{array}{c}
-0.0342 \\
0.3360 \\
-0.0198 \\
0.0010 \\
-0.3437 \\
-0.0099 \\
-0.0736 \\
-0.2748
\end{array}\right] .
$$

The sensitivity with respect to $\Delta P_{1}$ is zero. Thus, in terms of the three generator buses, we see that the most effective generator pair to redispatch is given by generators at bus 3 (the largest value) and 2 (the smallest value, most negative). Thus, the redispatch problem is formulated exactly as the power flow problem, except that additional variables are added for injections at buses 3 and 2. The resulting equation structure is therefore the same as previously, except that now we have an additional column to the Jacobian. In addition, a new equation specifying the active power flow has been added to the problem. During the first iteration of the new reformulated problem, the Jacobian matrix looks like

$$
\left[\begin{array}{lll}
\mathbf{H} & \mathbf{N} \\
\mathbf{M} & \mathbf{L}
\end{array}\right] \quad\left[\begin{array}{c}
-1 \\
1 \\
0 \\
0 \\
0 \\
0 \\
0 \\
0
\end{array}\right] \times\left[\begin{array}{l}
\Delta \theta_{2} \\
\Delta \theta_{3} \\
\Delta \theta_{4} \\
\Delta \theta_{5} \\
\Delta \theta_{6} \\
\Delta V_{4} \\
\Delta V_{5} \\
\Delta V_{6} \\
P^{\mathrm{r}}
\end{array}\right]=\left[\begin{array}{l}
0 \\
0 \\
0 \\
0 \\
0 \\
0 \\
0 \\
0 \\
3.5
\end{array}\right] .
$$


At this point, this linearized one iteration solution using this expanded Jacobian yields a new approximate solution to the original problem. In particular, the proposed redispatch value is equal to -9.44 . If a new power flow is solved with these new redispatch generation values, the solution for the new flow becomes 39.97 MW within any additional iterations needed.

\section{Relieving flow limits by phase shifter adjustments}

The phase-shifting transformer is a power system device that permits changes of complex transformation ratio (the ratio between primary and secondary complex voltages) and thus allowing the changes in voltage magnitudes as well as phase angles. By changing voltage magnitudes and phase angles it also changes flows over transmission lines (not only on the line where the device is installed). The problem here is to determine phase-shifting transformer most appropriate to handle limiting flow on a particular transmission line. As in the proceeding case, there are several options for the use of flow limit relief by means of phase shifters and these options include:

- The user designates the phase shifter (if any) to be used to relieve a particular flow limit.

- The phase shifter used to relieve the flow limit is selected as the "most effective" on a per-degree basis.

- The phase shifter used to relieve the flow limit is selected as the one with the widest available range of adjustment capability.

\subsection{Finding the most effective phase shifter}

Determining the effect of a phase shifter angle on a flow requires that the phase shifter angles $\alpha$ be included as explicit variables in the power flow formulation. In order to do this, it is better to use a polar formulation for the admittance matrix when considering taps. Thus, a slightly different but equivalent set of equations to those used so far can be used to characterize the power flow problem:

$$
\begin{aligned}
P_{i} & =V_{i} \sum_{j=1}^{n} V_{j} Y_{i j} \cos \left(\theta_{i j}-\gamma_{i j}\right), \\
Q_{i} & =V_{i} \sum_{j=1}^{n} V_{j} Y_{i j} \sin \left(\theta_{i j}-\gamma_{i j}\right) .
\end{aligned}
$$

Here $Y_{i j}$ denotes the magnitude of the corresponding entry of the nodal admittance matrix (which itself could incorporate the tap magnitude position as a variable, but this is not considered here), and $\gamma_{i j}$ denotes the phase angle of the corresponding entry of the nodal admittance matrix. Specifically, now, the phase angle of a phase shifting transformer becomes simply an adder to the angle of entry $i, j$ of the nodal admittance matrix, can becomes subtracted form entry $j, i$ of the same matrix. No other entries or values are affected.

To determine the impact of a phase shift on a flow (assuming all power injections remain constant), it is first necessary to determine the impact of a phase shifter adjustment on the angles and magnitudes of the voltages (assuming all injections remain constant, except of course for the slack bus active power injections). This can be accomplished by solving the following set of equations:

$$
\mathbf{J}\left[\begin{array}{c}
\Delta \boldsymbol{\theta} \\
\boldsymbol{\Delta} \mathbf{V}
\end{array}\right]=-\mathbf{J}^{\alpha} \Delta \boldsymbol{\alpha} .
$$

Here $\mathbf{J}$ is the ordinary power flow Jacobian, and $\mathbf{J}^{\alpha}$ is the Jacobian with respect to phase shifter angles. That is, it is the derivative of every bus power balance equation with respect to all the adjustable phase shifter angles. Only those buses that are directly connected to an active phase shifter are considered. For purpose of the analysis in this section, the phase shifters are considered one at the time. That is, the corresponding matrix $\mathbf{J}^{\alpha}$ is actually a vector, and the vector $\Delta \alpha$ is a scaler for each case of interest. The combined vector $\mathbf{J}^{\alpha} \Delta \boldsymbol{\alpha}$ is generally quite sparse. 
The determination of the nonzero entries for $\mathbf{J}^{\alpha}$ is as follows. Only the from node $i$ and the to node $j$ of the phase shifter are affected, thus we only give the formulas for these nonzero entries, both for $\mathbf{H}$ terms and $\mathbf{M}$ terms:

$$
\begin{aligned}
& H_{i}^{\alpha}=V_{i} V_{j} Y_{i j} \sin \left(\theta_{i j}-\gamma_{i j}\right), \\
& N_{j}^{\alpha}=-V_{i} V_{j} Y_{i j} \sin \left(\theta_{i j}-\gamma_{i j}\right), \\
& M_{i}^{\alpha}=-V_{i} V_{j} Y_{i j} \cos \left(\theta_{i j}-\gamma_{i j}\right), \\
& L_{j}^{\alpha}=V_{i} V_{j} Y_{i j} \cos \left(\theta_{i j}-\gamma_{i j}\right) .
\end{aligned}
$$

The differences in sign are quite important, and so is the fact that there is no "diagonal entry" to either term. These equations can be restated back in a rectangular formulation as follows:

$$
\begin{aligned}
& H_{i}^{\alpha}=V_{i} V_{j} G_{i j} \sin \theta_{i j}-V_{i} V_{j} B_{i j} \cos \theta_{i j}, \\
& N_{j}^{\alpha}=-V_{i} V_{j} G_{i j} \sin \theta_{i j}+V_{i} V_{j} B_{i j} \cos \theta_{i j}, \\
& M_{i}^{\alpha}=V_{i} V_{j} G_{i j} \sin \theta_{i j}-V_{i} V_{j} B_{i j} \cos \theta_{i j}, \\
& L_{j}^{\alpha}=-V_{i} V_{j} G_{i j} \sin \theta_{i j}+V_{i} V_{j} B_{i j} \cos \theta_{i j} .
\end{aligned}
$$

Once the effect on $\Delta \boldsymbol{\theta}$ and $\boldsymbol{\Delta} \mathbf{V}$ are obtained for every phase shifter in the system, their impact on every flow can be determined from

$$
\Delta \mathbf{P}^{\mathrm{f}}=\mathbf{H}^{\mathrm{f}} \boldsymbol{\Delta} \boldsymbol{\theta}+\mathbf{N}^{\mathrm{f}} \boldsymbol{\Delta} \mathbf{V}+\mathbf{A}^{\mathrm{f}} \boldsymbol{\Delta} \boldsymbol{\alpha},
$$

where $\mathbf{A}^{\mathrm{f}}$ is the Jacobian (derivative) of the desired flow equation with respect to changes in its phase angle setting. Only those elements that have a phase shifter embedded have to worry about a nonzero entry in this term. For all other elements, the effect of the phase shifter adjustment is captured entirely by the $\Delta \boldsymbol{\theta}$ and $\Delta \mathbf{V}$ vectors.

For phase-shifting transformers, we consider the case of the tap-side (the side where the shifting device is installed) and the non-tap side of the phase shifter separately. For the case of the non-tap side of the phase shifter, the corresponding equations for the flow on the transformer are

$$
P_{j i}=V_{j}^{2} G_{i j}-\alpha G_{i j} V_{i} V_{j} \cos \left(\theta_{i j}+\alpha\right)+\alpha B_{i j} V_{i} V_{j} \sin \left(\theta_{i j}+\alpha\right) .
$$

And thus the Jacobian entries for $\mathbf{A}^{\mathrm{f}}$ are given by (for the effect of the phase angle alone)

$$
\frac{\partial P_{j i}}{\partial \alpha}=-G_{i j} V_{i} V_{j} \cos \left(\theta_{i j}+\alpha\right)+\alpha G_{i j} V_{i} V_{j} \sin \left(\theta_{i j}+\alpha\right)+B_{i j} V_{i} V_{j} \sin \left(\theta_{i j}+\alpha\right)+\alpha B_{i j} V_{i} V_{j} \cos \left(\theta_{i j}+\alpha\right) .
$$

For the case of the tap-side of the phase shifter, the corresponding equations for the flow on the transformer are

$$
P_{i j}=\alpha^{2} V_{i}^{2} G_{i j}-\alpha G_{i j} V_{i} V_{j} \cos \left(\theta_{i j}+\alpha\right)-\alpha B_{i j} V_{i} V_{j} \sin \left(\theta_{i j}+\alpha\right) .
$$

And thus the Jacobian entries for $\mathbf{A}^{\mathrm{f}}$ are given by (for the effect of the phase angle alone)

$$
\begin{aligned}
\frac{\partial P_{i j}}{\partial \alpha}= & 2 \alpha V_{i}^{2} G_{i j}-G_{i j} V_{i} V_{j} \cos \left(\theta_{i j}+\alpha\right)+\alpha G_{i j} V_{i} V_{j} \sin \left(\theta_{i j}+\alpha\right) \\
& -B_{i j} V_{i} V_{j} \sin \left(\theta_{i j}+\alpha\right)-\alpha B_{i j} V_{i} V_{j} \cos \left(\theta_{i j}+\alpha\right) .
\end{aligned}
$$

Once the impact of every phase shifter angle change on every flow of interest is determined, a sort procedure similar to the one above can be done. In this case, however, if we assume an unlimited control range in either direction, the best phase shifter choice is the one that shows the largest absolute value to its sensitivity. Once this has been determined the next step can be undertaken.

\subsection{Power flow with flow limit and phase shifter adjustment}

The power flow with a phase shifter adjusted to control a desired limiting flow can be solved by formulating an ordinary power flow problem, but adding to it one degree of freedom (one variable), the angle at the designated 
phase shifter. At the same time one additional equation is included, the equation that specifies the flow in the desired controlled line. The Jacobian that is used in the solution is the same as before except that it has a new column corresponding to the derivatives of the injections with respect to angle adjustments in the phase shifter. In addition, there is one additional equation that specifies the flow on the constrained element, and its Jacobian entries for its row are those for the flow Jacobian $\mathbf{J}_{\mathrm{f}}$. Whether this extra row also has a nonzero entry in the column that corresponds to the phase shifter angle control or not depends entirely on whether the flow is being controlled on the element itself or on some other element. The fundamentals are the same either way.

\subsection{A simple numerical example with phase shifters}

We again use the system described in Section 3.3 with same limits on transmission lines, but this time try to resolve limits by means of phase shifters installed in the lines as illustrated in Fig. 1. Sensitivities of the active power flow on the limited line to the angles of all three phase shifting transformers are

$$
\begin{aligned}
& \frac{\mathrm{d} P_{36}}{\mathrm{~d} \alpha_{1-4}}=0.1, \\
& \frac{\mathrm{d} P_{36}}{\mathrm{~d} \alpha_{2-3}}=-2.8, \\
& \frac{\mathrm{d} P_{36}}{\mathrm{~d} \alpha_{5-6}}=1.9 .
\end{aligned}
$$

We see that the most effective phase-shifting transformer in alleviation of the limit is the one installed in the line between the buses 2 and 3. Derivatives of the injections with respect to angle adjustment in the phase shifter are

$$
\begin{aligned}
\frac{\partial P_{3}}{\partial \alpha_{2-3}} & =\frac{\partial P_{36}}{\partial \alpha_{2-3}}=1.6, \\
\frac{\partial P_{6}}{\partial \alpha_{2-3}} & =\frac{\partial P_{63}}{\partial \alpha_{2-3}}=-1.6, \\
\frac{\partial Q_{6}}{\partial \alpha_{2-3}} & =\frac{\partial Q_{63}}{\partial \alpha_{2-3}}=-0.05 .
\end{aligned}
$$

Thus, the problem to determine the size of phase shift is formulated exactly as the power flow problem, except that an additional variable is added. The resulting equation structure is therefore the same as previously, except that now we have an additional column to the Jacobian. In addition, a new equation specifying the active power flow has been added to the problem. During the first iteration of the new reformulated problem, the Jacobian matrix looks like

$$
\left[\begin{array}{ccc}
\mathbf{H} & \mathbf{N} \\
\mathbf{M} & \mathbf{L}
\end{array}\right] \quad\left[\begin{array}{c}
0 \\
-1.6 \\
0 \\
0 \\
1.6 \\
0 \\
0 \\
-0.05
\end{array}\right] \times\left[\begin{array}{c}
\Delta \theta_{2} \\
\Delta \theta_{3} \\
\Delta \theta_{4} \\
\Delta \theta_{5} \\
\Delta \theta_{6} \\
\Delta V_{4} \\
\Delta V_{5} \\
\Delta V_{6} \\
\alpha_{2-3}
\end{array}\right]=\left[\begin{array}{c}
0 \\
0 \\
0 \\
0 \\
0 \\
0 \\
0 \\
0 \\
3.5
\end{array}\right] .
$$

This linearized one iteration solution yields a new approximate solution to the original problem and the value of phase angle of the phase shifting transformer in the line between buses 2 and 3 is $-0.105 \operatorname{rad}\left(-6^{\circ}\right)$. If a new power flow is solved, the solution for the new flow on the line becomes $39.89 \mathrm{MW}$ within any additional iterations needed. 


\section{Examples using a real power system}

We take advantage of the availability of the IEEE Common Data Format (CDF) [14] for recently introduced approximate model of the European interconnected system [14]. The model is tuned to study effects of cross-border trades and indeed additional work was needed to make this model of the European interconnection suitable to test the methodology developed in this paper (most notably in putting appropriate transmission line limits). We use "Winter 2002" system loading conditions (higher load values and expected limit violations) CDF data from [14]. The whole network is shown in Fig. 2. The network includes 1254 buses (out of which 378 are generation buses) and 1944 lines.

We focus on two parts of this huge interconnection: Belgian and Netherlands system, and Eastern part of Italian system (toward Austria and Slovenia). This choice is based on the fact that there are some initiatives in placing or activating phase-shifting transformers in these systems (primarily to facilitate cross-border transmissions, but in this paper we asses their capabilities within proposed methodology and do not focus on cross-border trades) $[15,16]$.

The results are summarized in Table 1.

We also borrow the names of buses from [14]. Observe that the limits we considered in Belgium-Netherlands system were not solved by phase shifters only (in fact we found for the limited lines that only one phase-shifting transformer is truly effective) and the problem is solved by combining phase shifter adjustment and generation redispatch (with priority on phase shifters to minimize the size of redispatch). Two redispatch options were used: most effective and cheapest. Two limits considered in Eastern part of Italian system were

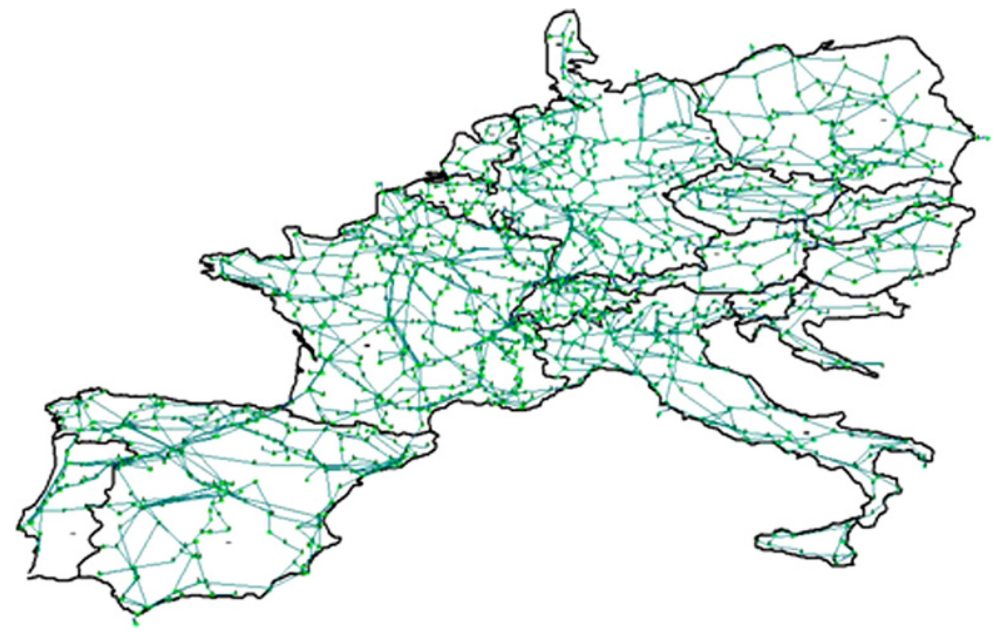

Fig. 2. European interconnected power system [14].

Table 1

The results using an approximate model of European interconnection

\begin{tabular}{|c|c|c|c|c|}
\hline \multirow[t]{2}{*}{ Line } & \multirow[t]{2}{*}{ MW (base case) } & \multirow[t]{2}{*}{ MW limit } & \multicolumn{2}{|l|}{ Correction } \\
\hline & & & PST & Gen. Resch. \\
\hline B_Zandvl-NL_Geert & 498.45 & 400 & $\begin{array}{l}\text { B_Zandvl-B-2 } \\
-4^{\circ}\end{array}$ & $\begin{array}{l}\text { "Most effective" } \\
\text { "INC" }=29.98 \mathrm{MW} \text { at B-2 } \\
\text { "DEC" }=-29.98 \mathrm{MW} \text { at NL_Borrs }\end{array}$ \\
\hline B_Zandvl-NL_Borrs & 439.93 & 400 & & $\begin{array}{l}\text { "Cheapest" } \\
\text { "INC" }=31.56 \mathrm{MW} \text { at B-2 } \\
\text { "DEC" }=-31.56 \mathrm{MW} \text { at NL_17 }\end{array}$ \\
\hline Fadalto-Vellai & 555.49 & 400 & $\begin{array}{l}\text { Fedalto-Caglian } \\
-7^{\circ}\end{array}$ & - \\
\hline Monfalc-Padricia & 151.67 & 120 & $\begin{array}{l}\text { Monfalc-Mantevia } \\
3^{\circ}\end{array}$ & \\
\hline
\end{tabular}


solved by the use of phase shifters only (as already indicated in [15] these transformers should solve line overloads in this part of the system).

\section{Conclusions}

Explicit specification of generation redispatch strategies and phase shifting transformer adjustments possible for flow limit enforcement have been presented in this paper. Sparse vectorized Newton-Raphson implementation power flow has been easily extended by explicit consideration of a flow limit equation in the set of system model equations. This new feature rendering the tool as a powerful and accurate helper for operating a power system within its security constraints. As for the line flow limits problem the operator is allowed to identify effective generator pair according to four different options, based on topology analysis, sensitivity studies, generator margins, or cost considerations as well as most effective phase-shifting transformers. Only active power line flow limit has been considered in the paper and including other two limits is straightforward. The presented features provide important new insights in the area focused in the paper. The results carried out with the help of a simple 6-bus test system and an approximate model of real-life European Interconnected Power System indicate that the methodology is effective.

\section{Appendix A. Line flow Jacobian matrix and extended power flow Jacobian}

In Section 3.1 the line flow Jacobian matrix $\mathbf{J}_{\mathrm{f}}$ has been introduced. The matrix has the form

$$
\mathbf{J}_{\mathrm{f}}=\left[\frac{\partial \mathbf{l}_{L}}{\partial \boldsymbol{\theta}}, \frac{\partial \mathbf{l}_{L}}{\partial \mathbf{V}}\right]
$$

where $l$ denotes generic line flow equation, and $L$ denotes the number of two terminal lines in the system. Depending of the limit considered, $l$ becomes $I_{\mathrm{f}}^{2}, P_{\mathrm{f}}$, or $S_{\mathrm{f}}$. To calculate the elements of the matrix we introduce the next notation:

$\overline{\mathbf{V}}_{L} \quad$ vector of line voltages $\left(\overline{\mathbf{V}}_{L}=\mathbf{A}^{\mathrm{T}} \overline{\mathbf{V}}\right)$

$\overline{\mathbf{I}}_{L} \quad$ vector of injected current of line $\left(\overline{\mathbf{I}}_{L}=\overline{\mathbf{Y}}_{\mathrm{f}} \overline{\mathbf{V}}_{L}\right)$

$\mathbf{A}_{n \times 2 L}$ an associate relationship matrix

$\mathbf{Y}_{\mathrm{f}(2 L \times 2 L)}$ the primary admittance matrix in which the diagonal elements are small admittance matrix (2-port representation of branch and transformer)

- $\quad$ multiplication of two vectors in point wise

$N \quad$ number of buses

$L \quad$ number of two terminal lines in the system

The elements of the flow Jacobian matrix are calculated as follows:

$$
\begin{aligned}
& \frac{\partial \overline{\mathbf{V}}_{L}}{\partial \mathbf{V}}=\mathbf{A}^{\mathrm{T}} \frac{\partial \overline{\mathbf{V}}}{\partial \mathbf{V}}=\mathbf{A}^{\mathrm{T}} \operatorname{diag}\left(\mathrm{e}^{\mathrm{j} \boldsymbol{\theta}}\right), \\
& \frac{\partial \overline{\mathbf{V}}_{L}}{\partial \boldsymbol{\theta}}=\mathbf{A}^{\mathrm{T}} \frac{\partial \overline{\mathbf{V}}}{\partial \boldsymbol{\theta}}=\mathbf{A}^{\mathrm{T}} \operatorname{diag}(\mathrm{j} \mathbf{V}), \\
& \frac{\partial \overline{\mathbf{I}}_{L}}{\partial \mathbf{V}}=\overline{\mathbf{Y}} \frac{\partial \overline{\mathbf{V}}_{L}}{\partial \mathbf{V}}=\overline{\mathbf{Y}}_{\mathrm{f}} \mathbf{A}^{\mathrm{T}} \operatorname{diag}\left(\mathrm{e}^{\mathrm{j} \boldsymbol{\theta}}\right), \\
& \frac{\partial \overline{\mathbf{I}}_{L}}{\partial \boldsymbol{\theta}}=\overline{\mathbf{Y}}_{\mathrm{f}} \frac{\partial \overline{\mathbf{V}}_{L}}{\partial \boldsymbol{\theta}}=\overline{\mathbf{Y}}_{\mathrm{f}} \mathbf{A}^{\mathrm{T}} \operatorname{diag}(\mathrm{j} \mathbf{V}), \\
& \frac{\partial\left(\operatorname{conj}\left(\overline{\mathbf{I}}_{L}\right)\right)}{\partial \mathbf{V}}=\operatorname{conj}\left(\overline{\mathbf{Y}}_{\mathrm{f}}\right) \mathbf{A}^{\mathrm{T}} \operatorname{diag}\left(\mathrm{e}^{-\mathrm{j} \boldsymbol{\theta}}\right), \\
& \frac{\partial\left(\operatorname{conj}\left(\overline{\mathbf{I}}_{L}\right)\right)}{\partial \boldsymbol{\theta}}=\operatorname{conj}\left(\overline{\mathbf{Y}}_{\mathrm{f}}\right) \mathbf{A}^{\mathrm{T}} \operatorname{diag}(-\mathrm{j} \mathbf{V}), \\
& \frac{\partial \mathbf{I}_{L}^{2}}{\partial \boldsymbol{\theta}}=\operatorname{diag}\left(\operatorname{conj}\left(\overline{\mathbf{I}}_{L}\right)\right) \frac{\partial \overline{\mathbf{I}}_{L}}{\partial \boldsymbol{\theta}}+\operatorname{diag}\left(\overline{\mathbf{I}}_{L}\right) \frac{\partial\left(\operatorname{conj}\left(\overline{\mathbf{I}}_{L}\right)\right)}{\partial \boldsymbol{\theta}},
\end{aligned}
$$




$$
\begin{aligned}
& \frac{\partial \mathbf{I}_{L}^{2}}{\partial \mathbf{V}}=\operatorname{diag}\left(\operatorname{conj}\left(\overline{\mathbf{I}}_{L}\right)\right) \frac{\partial \overline{\mathbf{I}}_{L}}{\partial \mathbf{V}}+\operatorname{diag}\left(\overline{\mathbf{I}}_{L}\right) \frac{\partial\left(\operatorname{conj}\left(\overline{\mathbf{I}}_{L}\right)\right)}{\partial \mathbf{V}}, \\
& \frac{\partial \mathbf{S}_{L}}{\partial \boldsymbol{\theta}}=\frac{\partial}{\partial \boldsymbol{\theta}}\left(\operatorname{conj}\left(\overline{\mathbf{Y}}_{\mathrm{f}}\right) \operatorname{conj}\left(\mathbf{V}_{L}\right) \bullet \mathbf{V}_{L}\right)=\operatorname{conj}\left(\overline{\mathbf{Y}}_{\mathrm{f}}\right)\left[\operatorname{diag}\left(\overline{\mathbf{V}}_{L}\right) \frac{\partial \overline{\mathbf{V}}_{L}}{\partial \boldsymbol{\theta}}+\operatorname{diag}\left(\operatorname{conj}\left(\overline{\mathbf{V}}_{L}\right) \frac{\partial\left(\operatorname{conj}\left(\overline{\mathbf{V}}_{L}\right)\right)}{\partial \boldsymbol{\theta}}\right],\right. \\
& \frac{\partial \mathbf{S}_{L}}{\partial \mathbf{V}}=\frac{\partial}{\partial \mathbf{V}}\left(\operatorname{conj}\left(\overline{\mathbf{Y}}_{\mathrm{f}}\right) \operatorname{conj}\left(\mathbf{V}_{L}\right) \bullet \mathbf{V}_{L}\right)=\operatorname{conj}\left(\overline{\mathbf{Y}}_{\mathrm{f}}\right)\left[\operatorname{diag}\left(\overline{\mathbf{V}}_{L}\right) \frac{\partial \overline{\mathbf{V}}_{L}}{\partial \mathbf{V}}+\operatorname{diag}\left(\operatorname{conj}\left(\overline{\mathbf{V}}_{L}\right) \frac{\partial\left(\operatorname{conj}\left(\overline{\mathbf{V}}_{L}\right)\right)}{\partial \mathbf{V}}\right],\right.
\end{aligned}
$$

where: $\mathrm{e}^{\mathrm{j} \theta}=\frac{\overline{\mathbf{v}}}{\mathrm{V}}$ point wisely.

$$
\mathbf{F F 1}=\mathbf{e}_{1}^{\mathrm{T}} \mathbf{J}_{\mathrm{f}},
$$

where $\mathbf{e}_{1}$ is the vector with all entries equal to zero but one corresponding to the limited line equal to 1

$$
\mathbf{s}^{\mathrm{g}}=\left(\mathbf{F F 1} \cdot \mathbf{F F 1} 1^{\mathrm{T}}\right)^{-1} \mathbf{J} \cdot \mathbf{F F 1} \mathbf{1}^{\mathrm{T}} .
$$

Let $M$ generators be assigned to participate in flow limit handling. Vector k1 contains $M$ nonzero elements. Nonzero value corresponding to $i$ th 'INC' generator is calculated by

$$
k 1_{i}=\frac{F D F_{i}}{\sum_{j \in \mathrm{INC}^{\prime}} F D F_{j}}
$$

and for corresponding 'DEC' generator

$$
k 1_{n}=-\frac{F D F_{n}}{\sum_{j \in{ }^{\prime} \mathrm{DEC}} F D F_{j}} .
$$

$F D F s$ are sensitivities taken from $\mathbf{s}^{\mathrm{g}}$.

\section{References}

[1] A.J. Wood, B.F. Wollenberg, Power Generation, Operation and Control, Wiley-Interscience, 1996.

[2] F.L. Alvarado, Solving power flow problems with a Matlab implementation of the power system applications data dictionary, Decision Support Systems 30 (3) (2001) 243-254.

[3] J. Mahseredijan, F. Alvarado, G. Rogers, W. Long, MATLAB's power for power systems, IEEE Computer Applications in Power (2001) 13-19.

[4] M. Glavic, F. Alvarado, Interactions among limits during maximum loadability and transfer capability determination, in: Electric Power Transfer Capability: Concepts, Applications, Sensitivity, Uncertainty, PSERC Report 01-34, University of Wisconsin, Madison, USA, 2001. [Online]. Available from: <www.pserc.cornell.edu/tcc $>$.

[5] T.K.P. Medicherla, R. Billinton, M.S. Sachdev, Generation rescheduling and load shedding to alleviate line overloads - analysis, IEEE Transactions on PAS 98 (6) (1979) 1876-1883.

[6] Z.X. Han, Phase shifter and power flow control, IEEE Transactions on PAS 101 (10) (1982) 3790-3795.

[7] N. Srinavasan, K.S. Prakasa Rao, C.S. Indulkar, S.S. Venkata, On-line computation of phase shifter distribution factors and lineload alleviation, IEEE Transactions on PAS 104 (7) (1985) 1656-1662.

[8] P.R. Bijwe, D.P. Kothari, L.D. Arya, Alleviation of line overloads and voltage violations by corrective rescheduling, IEE Proceedings-C 140 (4) (1993) 249-255.

[9] A. Mohamed, G.B. Jasmon, Realistic power security algorithm, IEE Proceedings-C 135 (2) (1988) 98-106.

[10] C.R. Fuerte-Esquivel, E. Acha, A Newton-type algorithm for the control of power flow in electric power networks, IEEE Transactions on Power Systems 12 (4) (1997) 1474-1480.

[11] B.K. Talukdar, A.K. Sinha, S. Mukhopadhyay, A. Bose, A computationally simple method for cost-efficient generation rescheduling and load shedding for congestion management, International Journal of Electric Power and Energy Systems 27 (2005) 379-388.

[12] M. Glavic, I. Dzafic, S. Tesnjak, Handling flow limits during maximum loadability determination, in: Mediterranean Electrotechnical Conference, MELECON 2004, Dubrovnik, Croatia, Paper 1060, 2004.

[13] J.A. Momoh, J.Z. Zhu, G.D. Boswell, S. Hoffman, Power system security enhancement by OPF with phase shifter, IEEE Transactions on Power Systems 16 (2) (2001) 287-293.

[14] Q. Zhou, J.W. Bialek, Approximate model of european interconnected power system as a benchmark system to study effects of crossborder trades, IEEE Transactions on Power Systems 20 (2) (2005) 782-788.

[15] P. Bresesti, M. Sforna, V. Allegranza, D. Canever, R. Vialati, Application of phase shifting transformers for a secure and efficient operation of the interconnection corridors, in: IEEE-PES General Meeting, Denver, Colorado, 2004, pp. 1192-1197.

[16] J. Verboomen, D. Van Hertem, P. Schavemaker, W. Kling, R. Belmans, Phase shifting transformers: principles and applications, in: International Conference on Future Power Systems, Amsterdam, The Netherlands, 2005. ISBN 90-78205-01-6. 